\title{
A Temperature Based Automatic Power Controller for Electrical Devices
}

\author{
Md. Murshadul Hoque ${ }^{1}$ and Shariful Alam ${ }^{2}$ \\ ${ }^{1}$ Department of Applied Physics, Electronics and Communication Engineering, \\ University of Chittagong, Bangladesh \\ ${ }^{2}$ Department of Biomedical Engineering, University of Malaya, Malaysia \\ m.hoque@cu.ac.bd',suzon_01cse@yahoo.com ${ }^{2}$
}

\begin{abstract}
In this paper, a controller is proposed to design in such a way that it can be able to control the input voltage as well as power to any load or electrical device not only manually i.e. through the computer but also automatically i.e. based on temperature. We propose this design for the reason that a part of the electrical appliances are needed to control the input power for getting the desired output from those which depend on the variation of temperature. Sometimes equipments can be needed manual control. For this purpose, computer controlling is also inserted here rather than supplying manpower. In this design, the voltage control technique is used to control the power to the load. Here, the output of the controller will vary on the sense that the variation of the surrounding temperature selects the relay which is connected to regulating coil to control the output voltage as well as power. The main specialty of this research is to maintain enhanced precision and hysteresis without harmonics in output variation.
\end{abstract}

Keywords: Automatic Power Controller, Temperature, Computer Control, Harmonics, Precision, Hysteresis

\section{Introduction}

In the modern age, we are trying to communicate with the outside of the world within a very short duration as much as possible. In such manner, we are always not only to deal with the persons to fulfill our correspondences but also to control the electrical and electronic machines, appliances, equipments etc to meet our daily requirements that supports for comforts. The existing control system can be done manually by supplying the proper manpower which becomes time \& cost ineffective, disgusting and unsuitable as well. So the necessity of automatic controlling is important.

The most existing automatic control systems [1-5] are lagging to get precise and smooth variation of output, and proper hysteresis. Otherwise, some are used to behave manually which is much more hazardous. All of the control systems, those are commercially available, are mostly integrated to the high costly and sophisticated appliances and equipments. That deprives the people to use this type of control systems to their designs and daily necessary devices and appliances.

Our main objective is to design an independent control system which responses on the ambience conditions. The proposed system is more reliable, easy to handle, cost effective that overcome the drawbacks of existing systems. This system provides the variation in output 
corresponding to the change of surrounding temperature. It is more flexible, compatible and adjustable to any electrical and electronic devices.

The rest of this paper is organized as follows. In Section 2, we introduce the design overview of our proposed system. In Section 3, the procedure of hardware design and circuit operation and in Section 4, computer controlled operation with parallel port are illustrated. The performance analysis is presented in Section 5, and finally we conclude this paper in conclusion section.

\section{Design Overview}

In this design, a precise centigrade temperature Sensor, LM35 [6] senses the temperature and its output are fed to an amplifier to amplify. The output of the amplifier is compared with a reference voltage in comparator and the output is connected with the relay by a switch [710], which makes the relay contact close. For different temperature different relay operates. So, a voltage appears across the load for a specific tap of the regulating coil $[7,11]$. The data cable is connected to the switch and the parallel port of computer. Data can be sent to the relay to operate through parallel port. Switch is used to distinguish how relay will operate. If the switch is "ON" then relay will operate depending on data or instructions that found from computer where, the switch is "OFF" then relay will operate depending temperature. Different relay is connected with different tapping of the regulating coil which controls the variation in the input voltage or power to the load [7, 12]. The proposed controlling unit which is responsible with the variation of surrounding temperature automatically as well as computer based. The block diagram (Figure 1) depicts the whole design.

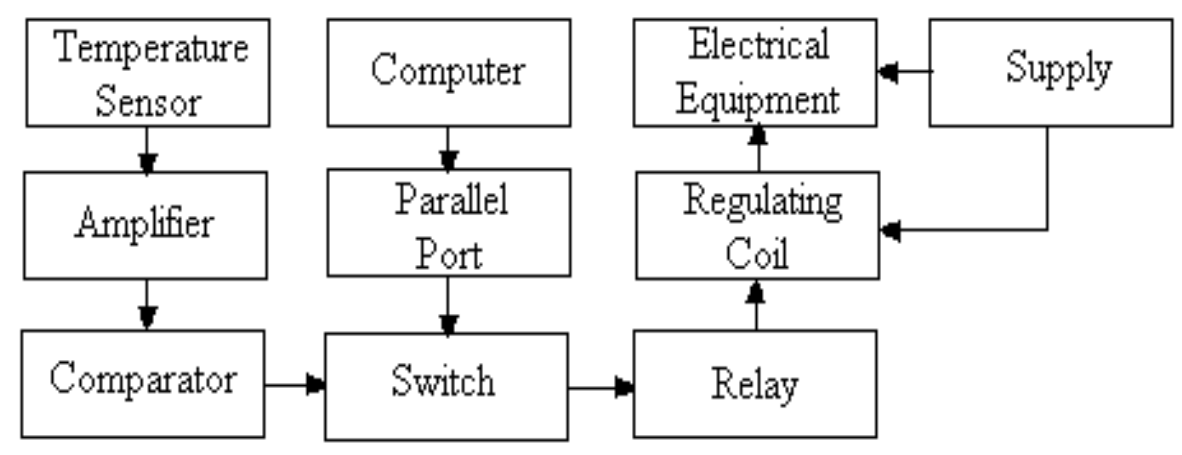

Figure 1. Block diagram of Electrical Equipment Controller

\section{Procedure of the Hardware Design and Circuit Operation}

Here, the output of this controller (i.e., the input to the controlled equipment) will vary in such a way that the variation of the output of the regulating coil is controlled by the switching of relays where, the relays are operated with the automatic response of the temperature sensor which depends on the changes of the surrounding temperature or on the computer program. The output of the temperature sensor is to be compared with a reference voltage level via an amplifier in the comparator [7-10] to operate the specific relay. In this work, four steps regulation is shown. It is important to be clear, here, that the selection of the controlling system either automatic or manual is functioned by the maintaining the position of the selection switch in "ON" stage or "OFF" stage. 
The Figure 2 below shows the full circuit with discrete components of this design. It is considered, here, as a temperature sensor LM35 which responses linearly with the temperature [6]. Here, the control circuit requires a 5V DC supply and the controller have to be connected in series with the equipment.

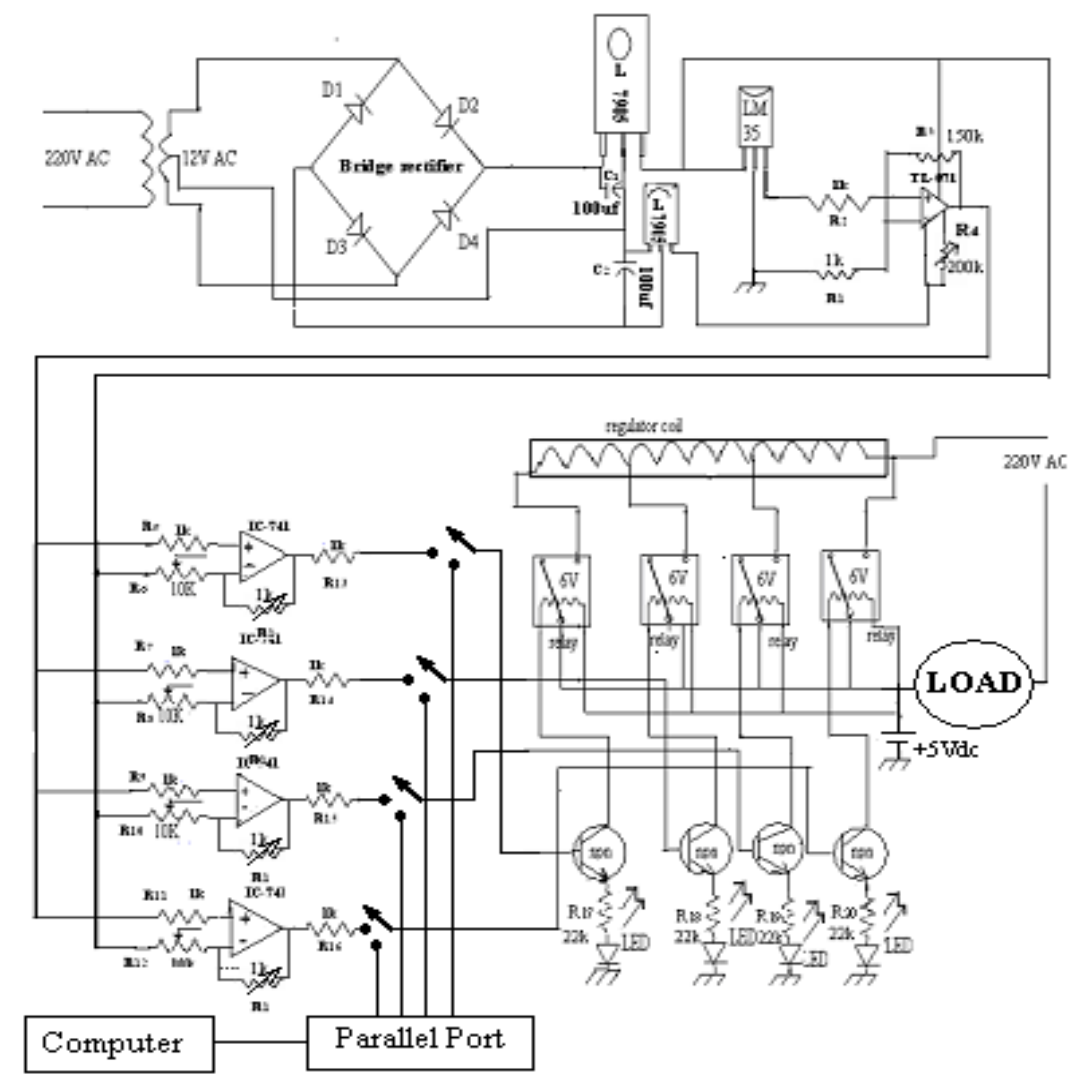

Figure 2. Circuit Diagram of the design

The output of the thermal sensor is varied for $10 \mathrm{mV} /{ }^{\circ} \mathrm{C}$ [6]. So, for a certain temperature, the thermal sensor gives an output which is amplified by an Op-Amp IC TL071. Then it is fed to inputs of the four comparators, IC 741 to be compared with a reference voltage level. To get the different level of reference voltages, the reference inputs of comparators are adjusted by varying the resistance. So that, these comparators generate a high output at a time for a certain change of temperature within maximum to minimum. The feedback networks of comparators maintain the proper hysteresis to switching and selection of the taps of the regulating coil while the output will change in back and forth. These outputs are connected to the relays through the switches. When a switch is turned on, the current flows through the relay coil and the contact is closed. For a specific temperature, comparators give high output in such a way that will in turn control relay to select the corresponding tap of regulating coil which is attached with the load in series. Hence, for different temperature, different voltage will appear across the load and power as well. 


\section{Computer Controlled Operation with Parallel Port}

For computer based communication, the parallel port [13] is mostly utilized which contains generally 25 pins of 8 pins for data, 5 pins for status and 4 pins for control. The hardware design of control system is directly connected to the computer via the parallel port. A controlling software [14] is used to interface with the hardware and computer where the layout of that is shown in Figure 3 as control basis. Here, it is shown that the different radio buttons are for different step of desire output variations. When a radio button is selected, the other buttons automatically are deselected, which produces high output to the parallel port to control the specific relay. The control stage can be increased by adapting a hardware set with software modification.

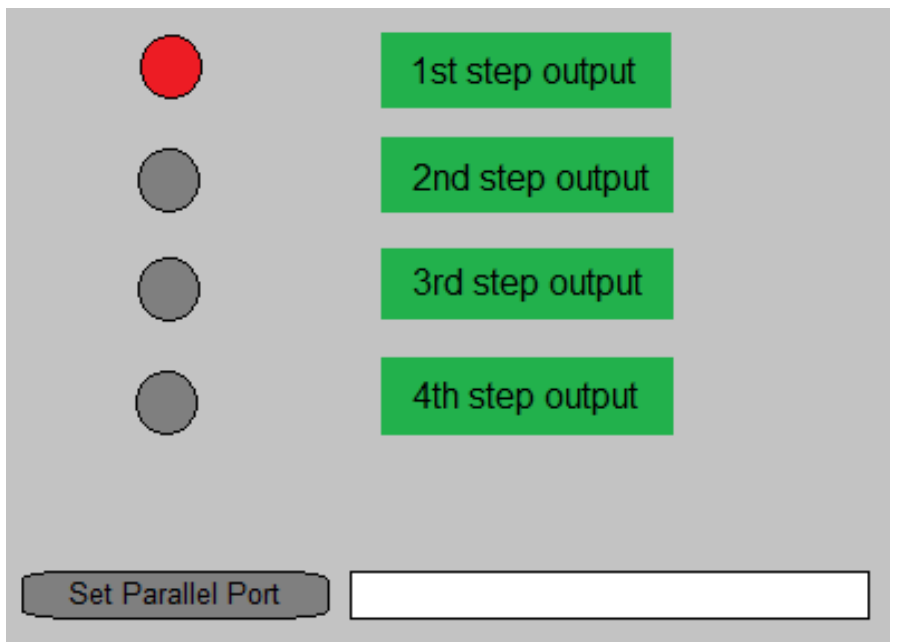

Figure 3. The layout of computer controlled interface

\section{Performance Analysis}

This design has been applied to an induction motor of an electrical fan as a load which is operated at maximum speed of $265 \mathrm{rpm}$ [7]. Here, the performance of the control unit is considered by the relay operation table, timing diagram and response curves. At this point, the speed of the induction motor has varied in accordance with the change of temperature.

The Table 1 shows the different relays' ON-OFF conditions and the resultant speeds. In this analysis, it is clearly observed that when relays are activated relatively successive to each other and the input power also varies which is investigated by the variation of speed of the load.

Table 1. Tabular representation of performance

\begin{tabular}{|c|c|c|c|c|}
\hline Relay-1 & Relay-2 & Relay-3 & Relay-4 & Speed(rpm) \\
\hline Off & Off & Off & Off & 0 \\
\hline ON & Off & Off & Off & 130 \\
\hline ON & ON & Off & Off & 205 \\
\hline ON & ON & ON & Off & 230 \\
\hline ON & ON & ON & ON & 265 \\
\hline
\end{tabular}


The output signals of parallel port, when the device is controlled by computer, are shown in the timing diagram (Figure 4). Here, signals have been found in such a way that when any relay is "ON" i.e. the high signal (logic 1) from the parallel port is fed to that relay, the other relays become "OFF" i.e. the signals for those relays are low (logic 0 ).

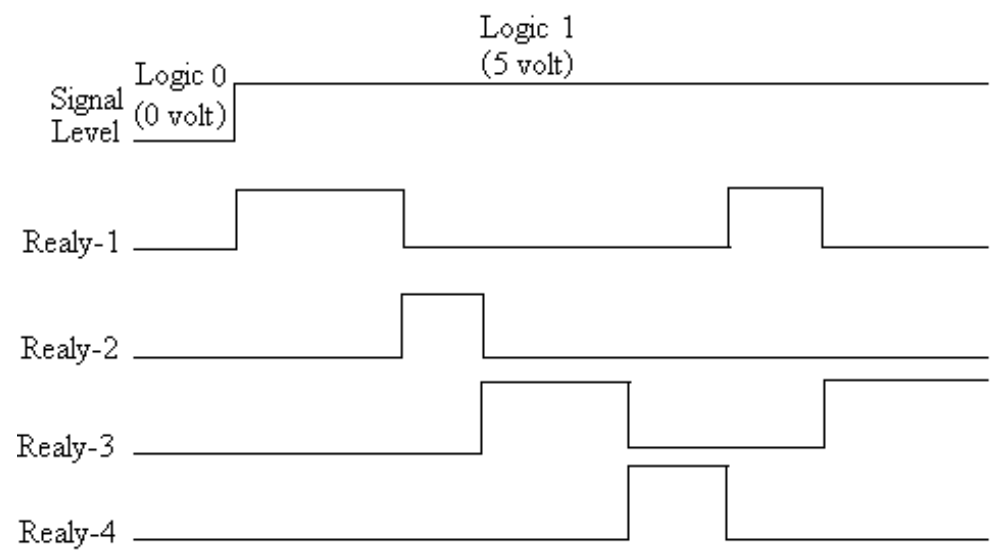

Figure 4. Timing diagram for relays during computer controlled

The variation of terminal voltage and speed of the induction motor with the temperature is given in Table 2.

Table 2. Recorded data of various speeds and terminal voltages of typical load corresponding to the different temperature

\begin{tabular}{|c|c|c|}
\hline Temperature $\left({ }^{\circ} \mathbf{C}\right)$ & Speed (rpm) & Voltage (Volts) \\
\hline 25 & 0 & 0 \\
\hline 27 & 130 & 195 \\
\hline 29 & 203 & 215 \\
\hline 30 & 230 & 218 \\
\hline 32 & 265 & 223 \\
\hline
\end{tabular}

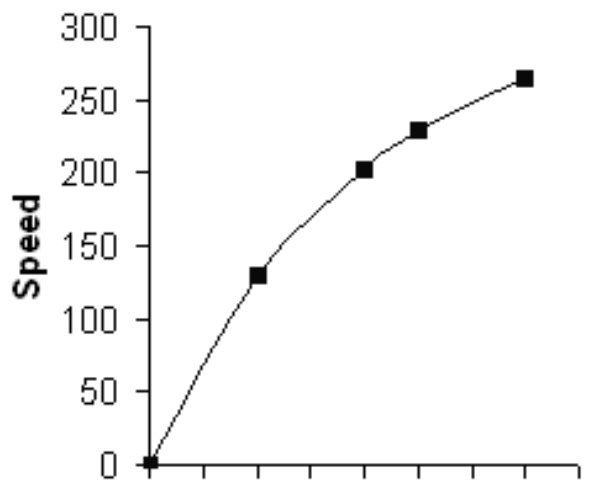

252627282930313233

Temperature

Figure 5. Speed versus Temperature Curve 


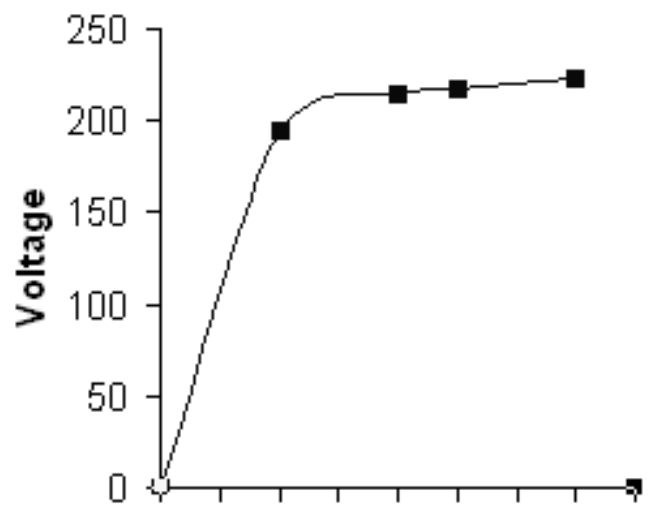

252627282930313233

Temperature

Figure 6. Voltage versus Temperature Curve

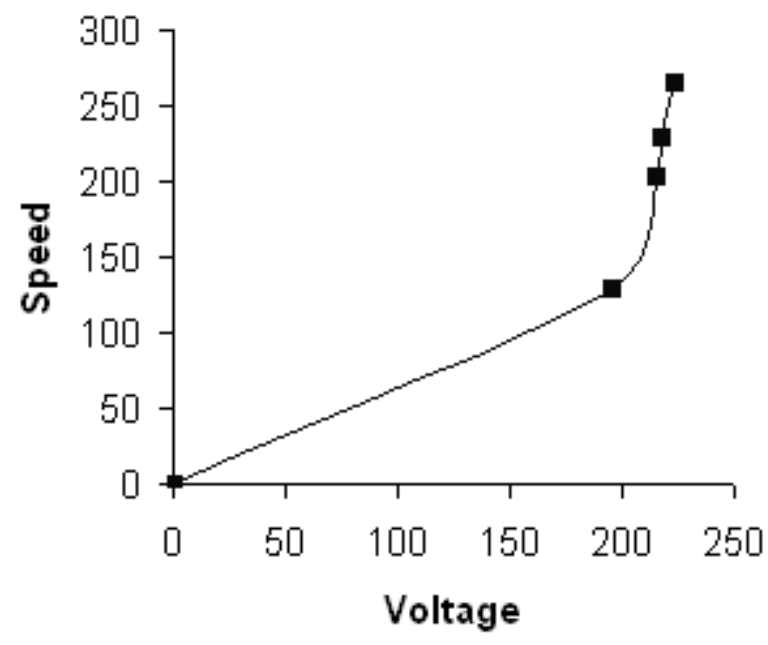

Figure 7. Speed versus Voltage Curve

In Figure 5, it is shown that the speed of fan has no rotation at temperature $25^{\circ} \mathrm{C}$ and then the speed of fan is increasing with the increase of temperature. Again, in Figure 6, it is shown that at $25^{\circ} \mathrm{C}$ temperature the voltage is zero and then voltage is increasing linearly corresponding to the increase of temperature. Similarly Figure 7 shows that the speed of fan varies in linear with the variation of voltage.

\section{Conclusion}

This idea of proprietary control unit is implicated with any systems of electrical and electronics in a compatible and high efficient manner to lead the smart controlled world. In this paper, it has been tried to present the best work for the design of this controller. From the above discussion and design, it has been proved that the automatic electrical equipment controller is to be designed in this way which depends on the variation of the ambient or surrounding temperature of the hall of equipment is used. There is no harmonics in the controlled input to the load or device due to the use of voltage control technique [7]. This 
controller responds not only on the change of temperature but also on manual operation that is performed through the computer. When the controller is operated through the instructions via computer, the instructions are instructed by the user where the user thinks what will be the output of controller. In this design, one suggestion will be introduced here for getting the more smooth output response of the controller that is to insert more relays with various taps of regulation coil.

\section{References}

[1] T. Kim, H. Lee and Y. Chung, "Advanced Universal Remote Controller for Home Automation and Security”, IEEE Transactions on Consumer Electronics, vol. 56, no. 4, (2010), pp. 2537-2542.

[2] S. M. M. R. Al-Arif, K. M. Kadir, M. A. Morshed, A. H. M. I. Ferdous, M. M. Alam and M. S. Haque, "Energy Saving by Limiting Standby Power Loss and Using Smart Power Control", Information Management, Innovation Management and Industrial Engineering (ICIII) International Conference, (2011) November 26-27, pp. 496 - 499; Shenzhen, China.

[3] C. -H. Lien, H. -C. Chen, Y. -W. Bai and M. -B. Lin, "Power Monitoring and Control for Electric Home Appliances Based on Power Line Communication", IEEE International Instrumentation and Measurement Technology Conference, (2008) May 12-15; Victoria, Canada.

[4] A. Petronilho and M. H. Terra, "Automatic control applied to home appliances, Control Applications (CCA)", IEEE International Conference, (2010) September 8-10, pp. 1182 - 1187; Yokohama, Japan.

[5] I. Bianchi, P. M. Filho, J. Pinto and F. Sobrinho, "Power performance evaluation of an electric home fan with triac-based automatic speed control system", Fundamental and Applied Metrology, XIX IMEKO World Congress, (2009) September 6-11; Lisbon, Portugal.

[6] Precise Centigrade Temperature Sensor LM35, http://www.ti.com/lit/ds/symlink/lm35.pdf.

[7] B. L. Theraja and A. K. Theraja, "A text book of Electrical Technology: AC and DC machines", S. Chand \& Company Ltd. Publisher, New Delhi, (2005).

[8] A. P. Malvino and D. J. Bates, "Electronic principles", McGraw-Hill Publisher, USA, (2006).

[9] J. Milmen and C. C. halkias, "Integrated electronics: Analog and Digital Circuit and system", McGraw-Hill Publisher, USA, (2006).

[10] R. L. Boylestad and L. Nashelsky, "Electronic Devices and circuit theory", Pearson Prentice Hall, UK, (2006).

[11] G. K. Mithal, "Industrial Electronic”, Khanna Publisher, New Dilhi, (1992).

[12] M. M. Hoque and A. I. Mahmod, "An Improved Automatic Voltage Regulation System with Apposite Hysteresis and Immense Precision”, Chittagong University Journal of Science, vol. 33, (2010), pp. 21-33.

[13] B. B. Bray, “The Intel Microprocessors 8086/8088, 80186/80188, 80286/80288, 80386, 80486 Pentium and Pentium Pro Processor, architecture, Programming and Interfacing”, Prentice Hall, New Jersey, (1997).

[14] X. Zhang, J. Sun and L. Zhou, "Development of an Internet Home Automation System using Java and Dynamic DNS Service", Sixth International Conference on Parallel and Distributed Computing, Applications and Technologies, PDCAT'05 Proceedings of IEEE International Conference, (2005) December 05, pp. 537 539; Washington, DC, USA.

\section{Authors}

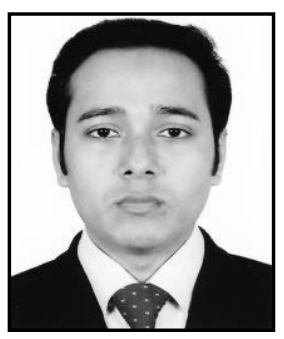

Md. Murshadul Hoque received B.Sc. (4years) degree in Electrical and Electronic Engineering from Chittagong University of Engineering and Technology (CUET), Bangladesh in 2006. Currently, he is working as an Assistant Professor in the Department of Applied Physics, Electronics and Communication Engineering at University of Chittagong, Bangladesh. His research interests, within power electronics, include integrated design, power system control \& automation, power conversion, alternative energy sources \& technologies and smart power grid. 


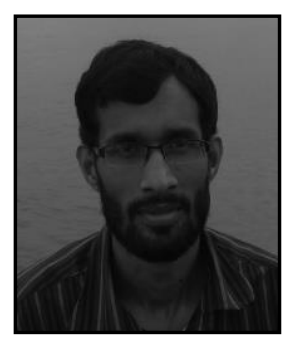

Shariful Alam received B.Sc. (4years) degree in Computer Science and Engineering from Chittagong University of Engineering and Technology (CUET), Bangladesh in 2006. Currently, he is pursuing his master degree at University of Malaya, Malaysia. His research interest includes signal processing and control system. 